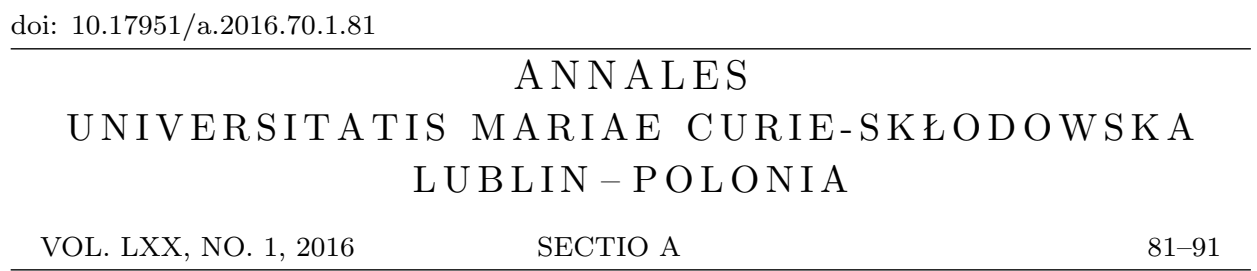

ANDRZEJ WALENDZIAK

\title{
On ideals of pseudo-BCH-algebras
}

\begin{abstract}
In this paper we introduce the notion of a disjoint union of pseudo-BCH-algebras and describe ideals in such algebras. We also investigate ideals of direct products of pseudo-BCH-algebras. Moreover, we establish conditions for the set of all minimal elements of a pseudo-BCH-algebra $\mathfrak{X}$ to be an ideal of $\mathfrak{X}$.
\end{abstract}

1. Introduction. In 1966, Y. Imai and K. Iséki ([11], [12]) introduced BCK- and BCI-algebras. In 1983, Q. P. Hu and X. Li ([10]) introduced $\mathrm{BCH}$-algebras. It is known that BCK- and BCI-algebras are contained in the class of $\mathrm{BCH}$-algebras.

In 2001, G. Georgescu and A. Iorgulescu ([9]) introduced pseudo-BCKalgebras as an extension of BCK-algebras. In 2008, W. A. Dudek and Y. B. Jun ([3]) introduced pseudo-BCI-algebras as a natural generalization of BCI-algebras and of pseudo-BCK-algebras. These algebras have also connections with other algebras of logic such as pseudo-MV-algebras and pseudo-BL-algebras defined by G. Georgescu and A. Iorgulescu (see [13]). Those algebras were investigated by several authors in [7], [8], [15] and [16]. Recently, A. Walendziak ([18]) introduced pseudo-BCH-algebras as an extension of $\mathrm{BCH}$-algebras and studied the set Cen $\mathfrak{X}$ of all minimal elements of a pseudo-BCH-algebra $\mathfrak{X}$, the so-called centre of $\mathfrak{X}$. He also considered ideals in pseudo-BCH-algebras and established a relationship between the ideals of a pseudo-BCH-algebra and the ideals of its centre.

2010 Mathematics Subject Classification. 03G25, 06F35.

Key words and phrases. (Pseudo-)BCK/BCI/BCH-algebra, disjoint union, ideal, centre. 
In this paper we introduce the notion of a disjoint union of pseudo-BCHalgebras and describe ideals in such algebras. We also investigate ideals of direct products of pseudo-BCH-algebras. Moreover, we establish conditions for the set Cen $\mathfrak{X}$ to be an ideal of a pseudo-BCH-algebra $\mathfrak{X}$.

2. Pseudo-BCH-algebras. We recall that an algebra $\mathfrak{X}=(X ; *, 0)$ of type $(2,0)$ is called a $B C H$-algebra if it satisfies the following axioms:

(BCH-1) $x * x=0$;

$(\mathrm{BCH}-2)(x * y) * z=(x * z) * y$;

(BCH-3) $x * y=y * x=0 \Longrightarrow x=y$.

A BCH-algebra $\mathfrak{X}$ is said to be a BCI-algebra if it satisfies the identity (BCI) $((x * y) *(x * z)) *(z * y)=0$.

A $B C K$-algebra is a BCI-algebra $\mathfrak{X}$ satisfying the law $0 * x=0$.

Definition 2.1 ([3]). A pseudo-BCI-algebra is a structure $\mathfrak{X}=(X ; \leq, *, \diamond, 0)$, where " $\leq$ " is a binary relation on the set $X$, “*" and " $\diamond$ " are binary operations on $X$ and " 0 " is an element of $X$, satisfying the axioms:

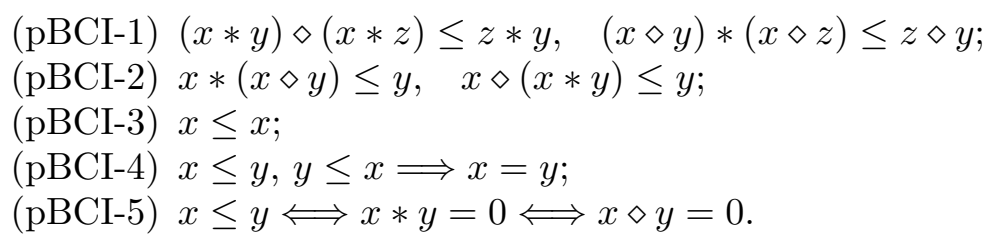

A pseudo-BCI-algebra $\mathfrak{X}$ is called a pseudo-BCK-algebra if it satisfies the identities

$(\mathrm{pBCK}) 0 * x=0 \diamond x=0$.

Definition $2.2([18])$. A pseudo-BCH-algebra is an algebra $\mathfrak{X}=(X ; *, \diamond, 0)$ of type $(2,2,0)$ satisfying the axioms:

$(\mathrm{pBCH}-1) x * x=x \diamond x=0$

$(\mathrm{pBCH}-2)(x * y) \diamond z=(x \diamond z) * y$;

$(\mathrm{pBCH}-3) \quad x * y=y \diamond x=0 \Longrightarrow x=y$;

$(\mathrm{pBCH}-4) x * y=0 \Longleftrightarrow x \diamond y=0$.

We define a binary relation $\leqslant$ on $X$ by

$$
x \leqslant y \Longleftrightarrow x * y=0 \Longleftrightarrow x \diamond y=0 .
$$

Throughout this paper $\mathfrak{X}$ will denote a pseudo-BCH-algebra.

Remark. Observe that if $(X ; *, 0)$ is a $\mathrm{BCH}$-algebra, then letting $x \diamond y:=$ $x * y$, produces a pseudo-BCH-algebra $(X ; *, \diamond, 0)$. Therefore, every $\mathrm{BCH}-$ algebra is a pseudo-BCH-algebra in a natural way. It is easy to see that if $(X ; *, \diamond, 0)$ is a pseudo-BCH-algebra, then $(X ; \diamond, *, 0)$ is also a pseudo-BCHalgebra. From Proposition 3.2 of [3] we conclude that if $(X ; \leq, *, \diamond, 0)$ is a pseudo-BCI-algebra, then $(X ; *, \diamond, 0)$ is a pseudo-BCH-algebra. 
Example $2.3([19])$. Let $(G ; \cdot, e)$ be a group. Define binary operations * and $\diamond$ on $G$ by

$$
a * b=a b^{-1} \quad \text { and } \quad a \diamond b=b^{-1} a
$$

for all $a, b \in G$. Then $\mathfrak{G}=(G ; *, \diamond, e)$ is a pseudo-BCH-algebra.

We say that a pseudo-BCH-algebra $\mathfrak{X}$ is proper if $* \neq \diamond$ and it is not a pseudo-BCI-algebra.

Remark. The class of all pseudo-BCH-algebras is a quasi-variety. Therefore, if $\left(\mathfrak{X}_{t}\right)_{t \in T}$ is an indexed family of pseudo-BCH-algebras, then the direct product $\mathfrak{X}=\prod_{t \in T} \mathfrak{X}_{t}$ is also a pseudo-BCH-algebra. In the case when at least one of $\mathfrak{X}_{t}$ is proper, then $\mathfrak{X}$ is proper.

Example 2.4. Let $X_{1}=\{0, a, b, c\}$. We define the binary operations $*_{1}$ and $\diamond_{1}$ on $X_{1}$ as follows:

\begin{tabular}{|c|c|c|c|c|c|c|c|c|c|c|}
\hline$*_{1}$ & 0 & $a$ & $b$ & $c$ & & $\diamond_{1}$ & 0 & $a$ & $b$ & $c$ \\
\hline 0 & 0 & 0 & 0 & 0 & & 0 & 0 & 0 & 0 & 0 \\
\hline$a$ & $a$ & 0 & $a$ & 0 & and & $a$ & $a$ & 0 & $a$ & 0 \\
\hline$b$ & $b$ & $b$ & 0 & 0 & & $b$ & $b$ & $b$ & 0 & 0 \\
\hline$c$ & $c$ & $b$ & $c$ & 0 & & $c$ & $c$ & $c$ & $a$ & 0 \\
\hline
\end{tabular}

On the set $X_{2}=\{0,1,2,3\}$ consider the operation $*_{2}$ given by the following table:

\begin{tabular}{c|cccc}
$*_{2}$ & 0 & 1 & 2 & 3 \\
\hline 0 & 0 & 0 & 0 & 0 \\
1 & 1 & 0 & 0 & 1 \\
2 & 2 & 2 & 0 & 0 \\
3 & 3 & 3 & 3 & 0
\end{tabular}

Then $\mathfrak{X}_{1}=\left(X_{1} ; *_{1}, \diamond_{1}, 0\right)$ and $\mathfrak{X}_{2}=\left(X_{2} ; *_{2}, *_{2}, 0\right)$ are pseudo-BCH-algebras (see [18]). Therefore, the direct product $\mathfrak{X}=\mathfrak{X}_{1} \times \mathfrak{X}_{2}$ is a (proper) pseudoBCH-algebra.

Let $\mathfrak{X}=(X ; *, \diamond, 0)$ be a pseudo-BCH-algebra satisfying (pBCK), and let $(G ; \cdot, e)$ be a group. Denote $Y=G-\{e\}$ and suppose that $X \cap Y=\emptyset$. Define the binary operations $*$ and $\diamond$ on $X \cup Y$ by

$$
x * y= \begin{cases}x * y & \text { if } x, y \in X \\ x y^{-1} & \text { if } x, y \in Y \text { and } x \neq y \\ 0 & \text { if } x, y \in Y \text { and } x=y \\ y^{-1} & \text { if } x \in X, y \in Y \\ x & \text { if } x \in Y, y \in X\end{cases}
$$


and

$$
x \diamond y= \begin{cases}x \diamond y & \text { if } x, y \in X \\ y^{-1} x & \text { if } x, y \in Y \text { and } x \neq y \\ 0 & \text { if } x, y \in Y \text { and } x=y \\ y^{-1} & \text { if } x \in X, y \in Y \\ x & \text { if } x \in Y, y \in X .\end{cases}
$$

Routine calculations give that $(X \cup Y ; *, \diamond, 0)$ is a pseudo-BCH-algebra; it is proper if $\mathfrak{X}$ is proper.

Example 2.5. Consider the set $X=\{0, a, b, c\}$ with the operation $*$ defined by the following table:

$$
\begin{array}{l|llll}
* & 0 & a & b & c \\
\hline 0 & 0 & 0 & 0 & 0 \\
a & a & 0 & c & c \\
b & b & 0 & 0 & b \\
c & c & 0 & 0 & 0
\end{array}
$$

Then $\mathfrak{X}=(X ; *, 0)$ is a BCH-algebra (see [10]). Let $\mathfrak{G}$ be the group of all permutations of $\{1,2,3\}$. We have $G=\{\imath, d, e, f, g, h\}$, where $\imath=(1), d=$ (12), $e=(13), f=(23), g=(123)$, and $h=(132)$. Applying (1) and (2) we obtain the following tables:

and

\begin{tabular}{l|lllllllll}
$*$ & 0 & $a$ & $b$ & $c$ & $d$ & $e$ & $f$ & $g$ & $h$ \\
\hline 0 & 0 & 0 & 0 & 0 & $d$ & $e$ & $f$ & $h$ & $g$ \\
$a$ & $a$ & 0 & $c$ & $c$ & $d$ & $e$ & $f$ & $h$ & $g$ \\
$b$ & $b$ & 0 & 0 & $b$ & $d$ & $e$ & $f$ & $h$ & $g$ \\
$c$ & $c$ & 0 & 0 & 0 & $d$ & $e$ & $f$ & $h$ & $g$ \\
$d$ & $d$ & $d$ & $d$ & $d$ & 0 & $h$ & $g$ & $e$ & $f$ \\
$e$ & $e$ & $e$ & $e$ & $e$ & $g$ & 0 & $h$ & $f$ & $d$ \\
$f$ & $f$ & $f$ & $f$ & $f$ & $h$ & $g$ & 0 & $d$ & $e$ \\
$g$ & $g$ & $g$ & $g$ & $g$ & $e$ & $f$ & $d$ & 0 & $h$ \\
$h$ & $h$ & $h$ & $h$ & $h$ & $f$ & $d$ & $e$ & $g$ & 0
\end{tabular}

\begin{tabular}{l|lllllllll}
$\diamond$ & 0 & $a$ & $b$ & $c$ & $d$ & $e$ & $f$ & $g$ & $h$ \\
\hline 0 & 0 & 0 & 0 & 0 & $d$ & $e$ & $f$ & $h$ & $g$ \\
$a$ & $a$ & 0 & $c$ & $c$ & $d$ & $e$ & $f$ & $h$ & $g$ \\
$b$ & $b$ & 0 & 0 & $b$ & $d$ & $e$ & $f$ & $h$ & $g$ \\
$c$ & $c$ & 0 & 0 & 0 & $d$ & $e$ & $f$ & $h$ & $g$ \\
$d$ & $d$ & $d$ & $d$ & $d$ & 0 & $h$ & $g$ & $f$ & $e$ \\
$e$ & $e$ & $e$ & $e$ & $e$ & $g$ & 0 & $h$ & $d$ & $f$ \\
$f$ & $f$ & $f$ & $f$ & $f$ & $h$ & $g$ & 0 & $e$ & $d$ \\
$g$ & $g$ & $g$ & $g$ & $g$ & $e$ & $f$ & $d$ & 0 & $g$ \\
$h$ & $h$ & $h$ & $h$ & $h$ & $f$ & $d$ & $e$ & $h$ & 0
\end{tabular}

Then $(\{0, a, b, c, d, e, f, g, h\} ; *, \diamond, 0)$ is a pseudo-BCH-algebra. Observe that it is proper. Indeed, $(b * c) \diamond(b * a)=b \diamond 0=b \nless c=a * c$. 
Let $T$ be any set and, for each $t \in T$, let $\mathfrak{X}_{t}=\left(X_{t} ; *_{t}, \diamond_{t}, 0\right)$ be a pseudoBCH-algebra satisfying (pBCK). Suppose that $X_{s} \cap X_{t}=\{0\}$ for $s \neq t$, $s, t \in T$. Set $X=\bigcup_{t \in T} X_{t}$ and define the binary operations $*$ and $\diamond$ on $X$ via

$$
x * y= \begin{cases}x *_{t} y & \text { if } x, y \in X_{t}, t \in T, \\ x & \text { if } x \in X_{s}, y \in X_{t}, s \neq t, s, t \in T,\end{cases}
$$

and

$$
x \diamond y= \begin{cases}x \diamond_{t} y & \text { if } x, y \in X_{t}, t \in T, \\ x & \text { if } x \in X_{s}, y \in X_{t}, s \neq t, s, t \in T .\end{cases}
$$

It is easy to check that $\mathfrak{X}=(X ; *, \diamond, 0)$ is a pseudo-BCH-algebra. Following the terminology for $\mathrm{BCH}$-algebras (see [1]), the algebra $\mathfrak{X}$ will be called the disjoint union of $\left(\mathfrak{X}_{t}\right)_{t \in T}$. We shall denote it by $\sum_{t \in T} \mathfrak{X}_{t}$.

Example 2.6. Let $\mathfrak{X}_{1}=\left(\{0, a, b, c\} ; *_{1}, \diamond_{1}, 0\right)$ be the pseudo-BCH-algebra from Example 2.4. Consider the set $X_{2}=\{0,1,2,3\}$ with the operation $*_{2}$ defined by the following table:

\begin{tabular}{c|cccc}
$*_{2}$ & 0 & 1 & 2 & 3 \\
\hline 0 & 0 & 0 & 0 & 0 \\
1 & 1 & 0 & 2 & 1 \\
2 & 2 & 0 & 0 & 2 \\
3 & 3 & 3 & 0 & 0
\end{tabular}

Routine calculations show that $\mathfrak{X}_{2}=\left(X_{2} ; *_{2}, *_{2}, 0\right)$ is a (pseudo)-BCHalgebra. Let $X=\{0, a, b, c, 1,2,3\}$. We define the binary operations *

\begin{tabular}{|c|c|c|c|c|c|c|c|c|c|c|c|c|c|c|c|}
\hline & 0 & $a$ & $b$ & $c$ & 1 & 2 & 3 & & $\diamond$ & 0 & $a$ & $b$ & $c$ & 1 & \\
\hline 0 & 0 & 0 & 0 & 0 & 0 & 0 & 0 & & 0 & 0 & 0 & 0 & 0 & 0 & ) \\
\hline$a$ & $a$ & 0 & $a$ & 0 & $a$ & $a$ & $a$ & & $a$ & $a$ & 0 & $a$ & 0 & $a$ & \\
\hline$b$ & $b$ & $b$ & 0 & 0 & $b$ & $b$ & $b$ & ond & $b$ & $b$ & $b$ & 0 & 0 & $b$ & \\
\hline$c$ & $c$ & $b$ & $c$ & 0 & $c$ & $c$ & $c$ & वाल & $c$ & $c$ & $c$ & $a$ & 0 & $c$ & f \\
\hline 1 & 1 & 1 & 1 & 1 & 0 & 2 & 1 & & 1 & 1 & 1 & 1 & 1 & 0 & 2 \\
\hline 2 & 2 & 2 & 2 & 2 & 0 & 0 & 2 & & 2 & 2 & 2 & 2 & 2 & 0 & ) \\
\hline 3 & 3 & 3 & 3 & 3 & 3 & 0 & 0 & & 3 & 3 & 3 & 3 & 3 & & \\
\hline
\end{tabular}
and $\diamond$ on $X$ as follows

It is clear that $\mathfrak{X}=(X ; *, \diamond, 0)$ is the disjoint union of $\mathfrak{X}_{1}$ and $\mathfrak{X}_{2}$. We have $(3 * 1) \diamond(3 * 2)=3 \diamond 0=3 \not 0=2 * 1$, and therefore $\mathfrak{X}$ is not a pseudo-BCI-algebra. Thus $\mathfrak{X}$ is a proper pseudo-BCH-algebra.

From [18] it follows that in any pseudo-BCH-algebra $\mathfrak{X}$ for all $x, y \in X$ we have:

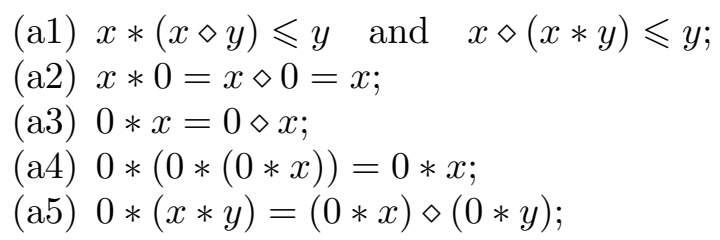


(a6) $0 *(x \diamond y)=(0 * x) *(0 * y)$.

Following the terminology of [18], the set $\{a \in X: a=0 *(0 * a)\}$ will be called the centre of $\mathfrak{X}$. W shall denote it by Cen $\mathfrak{X}$. By Proposition 4.1 of [18], Cen $\mathfrak{X}$ is the set of all minimal elements of $\mathfrak{X}$, that is,

$$
\text { Cen } \mathfrak{X}=\left\{a \in X: \forall_{x \in X}(x \leqslant a \Longrightarrow x=a)\right\} \text {. }
$$

By (a4),

$$
0 * x \in \operatorname{Cen} \mathfrak{X}
$$

for all $x \in \mathfrak{X}$.

Minimal elements (also called atoms) were investigated in $\mathrm{BCI} / \mathrm{BCH}-$ algebras ([17], [14]), pseudo-BCI-algebras ([7]), and in other algebras of logic (see for example [2], [4], and [5]).

Proposition 2.7 ([18]). Let $\mathfrak{X}$ be a pseudo-BCH-algebra, and let $a \in X$. Then the following conditions are equivalent:

(i) $a \in$ Cen $\mathfrak{X}$.

(ii) $a * x=0 *(x * a)$ for all $x \in X$.

(iii) $a \diamond x=0 *(x \diamond a)$ for all $x \in X$.

Proposition $2.8([18])$. Cen $\mathfrak{X}$ is a subalgebra of $\mathfrak{X}$.

\section{Ideals in pseudo-BCH-algebras.}

Definition 3.1. A subset $I$ of $X$ is called an ideal of $\mathfrak{X}$ if it satisfies for all $x, y \in X$,

(I1) $0 \in I$;

(I2) if $x * y \in I$ and $y \in I$, then $x \in I$.

We will denote by $\operatorname{Id}(\mathfrak{X})$ the set of all ideals of $\mathfrak{X}$. Obviously, $\{0\}, X \in$ $\operatorname{Id}(\mathfrak{X})$.

Proposition $3.2([18])$. Let $I$ be an ideal of $\mathfrak{X}$. For any $x, y \in X$, if $y \in I$ and $x \leqslant y$, then $x \in I$.

Proposition $3.3([18])$. Let $\mathfrak{X}$ be a pseudo-BCH-algebra and $I$ be a subset of $X$ satisfying (I1). Then $I$ is an ideal of $\mathfrak{X}$ if and only if for all $x, y \in X$, (I2') if $x \diamond y \in I$ and $y \in I$, then $x \in I$.

Example 3.4. Consider the pseudo-BCH-algebra $\mathfrak{G}$ given in Example 2.3. Let $a$ be an element of $G$. It is clear that $\left\{a^{n}: n \in \mathbb{N} \cup\{0\}\right\}$ is an ideal of $\mathfrak{G}$.

Example 3.5. Let $\mathfrak{X}_{1}=\left(\{0, a, b, c\} ; *_{1}, \diamond_{1}, 0\right)$ be the pseudo-BCH-algebra from Example 2.4. It is easy to check that $I_{1}=\{0\}, I_{2}=\{0, a\}, I_{3}=\{0, b\}$, and $I_{4}=\{0, a, b, c\}$ are ideals of $\mathfrak{X}_{1}$. Let $I$ be an ideal of $\mathfrak{X}_{1}$ and suppose that $c \in I$. Since $a *_{1} c=b *_{1} c=0 \in I$, (I2) shows that $a, b \in I$, and therefore $I=X_{1}$. Similarly, if $a, b \in I$, then $I=X_{1}$. $\operatorname{Thus} \operatorname{Id}\left(\mathfrak{X}_{1}\right)=\left\{I_{1}, I_{2}, I_{3}, I_{4}\right\}$. 
Theorem 3.6. Let $\mathfrak{X}$ be a pseudo-BCH-algebra and $I$ be a subset of $X$ containing 0 . The following statements are equivalent:

(i) $I$ is an ideal of $\mathfrak{X}$.

(ii) $x \in I, y \in X-I \Longrightarrow y * x \in X-I$.

(iii) $x \in I, y \in X-I \Longrightarrow y \diamond x \in X-I$.

Proof. (i) $\Longrightarrow$ (ii): Assume that $I$ is an ideal of $\mathfrak{X}$, let $x \in I$ and $y \in X-I$. If $y * x \in I$, then $y \in I$ by definition. Therefore $y * x \in X-I$.

(ii) $\Longrightarrow$ (i): To prove that $I \in \operatorname{Id}(\mathfrak{X})$, let $y * x \in I$ and $x \in I$. If $y \notin I$, then (ii) implies $y * x \in X-I$, a contradiction. Hence $y \in I$, which gives that $I$ is an ideal of $\mathfrak{X}$.

Thus we have (i) $\Longleftrightarrow$ (ii). The proof of the equivalence of (i) and (iii) is similar.

For any pseudo-BCH-algebra $\mathfrak{X}$, we set

$$
\mathrm{K}(\mathfrak{X})=\{x \in X: 0 \leqslant x\} .
$$

Proposition $3.7([18])$. Let $\mathfrak{X}_{1}$ and $\mathfrak{X}_{2}$ be pseudo-BCH-algebras. Then

$$
\mathrm{K}\left(\mathfrak{X}_{1} \times \mathfrak{X}_{2}\right)=\mathrm{K}\left(\mathfrak{X}_{1}\right) \times \mathrm{K}\left(\mathfrak{X}_{2}\right) .
$$

Observe that

$$
\text { Cen } \mathfrak{X} \cap \mathrm{K}(\mathfrak{X})=\{0\} .
$$

Indeed, $0 \in \operatorname{Cen} \mathfrak{X} \cap \mathrm{K}(\mathfrak{X})$ and if $x \in \operatorname{Cen} \mathfrak{X} \cap \mathrm{K}(\mathfrak{X})$, then $x=0 *(0 * x)=$ $0 * 0=0$.

\section{Theorem 3.8.}

(i) For any $t \in T$, let $I_{t}$ be an ideal of a pseudo-BCH-algebra $\left(X_{t} ; *_{t}, \circ_{t}, 0_{t}\right)$. Then $I:=\prod_{t \in T} I_{t}$ is an ideal of $\mathfrak{X}:=\prod_{t \in T} \mathfrak{X}_{t}$.

(ii) If $I$ is an ideal of $\mathfrak{X}$ such that $I \subseteq \mathrm{K}(\mathfrak{X})$, then $I_{t}:=\pi_{t}(I)$, where $\pi_{t}$ is the $t$-th projection of $\mathfrak{X}$ onto $\mathfrak{X}_{t}$, is an ideal of $\mathfrak{X}_{t}$, and $I \subseteq \prod_{t \in T} I_{t}$.

Proof. (i) The first part of the assertion is obvious.

(ii) The proof of this is similar to that of Theorem 5.1 .35 [6].

Proposition 3.9. Let $\mathfrak{X}_{1}$ and $\mathfrak{X}_{2}$ be pseudo-BCH-algebras satisfying the condition (pBCK). Then

$$
\operatorname{Id}\left(\mathfrak{X}_{1} \times \mathfrak{X}_{2}\right)=\operatorname{Id}\left(\mathfrak{X}_{1}\right) \times \operatorname{Id}\left(\mathfrak{X}_{2}\right) .
$$

Proof. Let $\mathfrak{X}=\mathfrak{X}_{1} \times \mathfrak{X}_{2}$ and $I \in \operatorname{Id}(\mathfrak{X})$. By Proposition 3.7, $\mathrm{K}(\mathfrak{X})=$ $\mathrm{K}\left(\mathfrak{X}_{1}\right) \times \mathrm{K}\left(\mathfrak{X}_{2}\right)=X_{1} \times X_{2}=X$, and therefore $I \subseteq \mathrm{K}(\mathfrak{X})$. From Theorem 3.8 (ii) it follows that $I \subseteq I_{1} \times I_{2}$, where $I_{1}=\pi_{1}(I), I_{2}=\pi_{2}(I)$. Let $a \in I_{1}$ and $b \in I_{2}$. There are $c \in X_{2}$ and $d \in X_{1}$ such that $(a, c),(d, b) \in I$. Since $(a, 0) \leqslant(a, c)$ and $(0, b) \leqslant(d, b)$, we conclude that $(a, 0),(0, b) \in I$. Observe that $(a, b) \in I$. Indeed, we have $(a, b) *(0, b)=(a, 0)$ and $(a, 0),(0, b) \in I$. From this $(a, b) \in I$. Therefore $I=I_{1} \times I_{2} \in \operatorname{Id}\left(\mathfrak{X}_{1}\right) \times \operatorname{Id}\left(\mathfrak{X}_{2}\right)$. 
Conversely, let $I=I_{1} \times I_{2}$, where $I_{1} \in \operatorname{Id}\left(\mathfrak{X}_{1}\right)$ and $I_{2} \in \operatorname{Id}\left(\mathfrak{X}_{2}\right)$. By Theorem 3.8 (i), $I$ is an ideal of $\mathfrak{X}$.

Example 3.10. Let $\mathfrak{X}=\mathfrak{X}_{1} \times \mathfrak{X}_{2}$ be the pseudo-BCH-algebra given in Example 2.4. We know that $\operatorname{Id}\left(\mathfrak{X}_{1}\right)=\left\{I_{1}, I_{2}, I_{3}, I_{4}\right\}$ where $I_{1}=\{0\}, I_{2}=$ $\{0, a\}, I_{3}=\{0, b\}$, and $I_{4}=X_{1}$ (see Example 3.5). It is easily seen that the only ideals of $\mathfrak{X}_{2}$ are the following subsets of $X_{2}: J_{1}=\{0\}, J_{2}=\{0,1\}$, $J_{3}=\{0,1,2\}$, and $J_{4}=X_{2}$. Then, by Proposition 3.9, $\operatorname{Id}(\mathfrak{X})=\left\{I_{m} \times J_{n}\right.$ : $m, n=1,2,3,4\}$.

Theorem 3.11. Let $\left(\mathfrak{X}_{t}\right)_{t \in T}$ be an indexed family of pseudo-BCH-algebras satisfying $(\mathrm{pBCK})$ and $\mathfrak{X}=\sum_{t \in T} \mathfrak{X}_{t}$. Let $I_{t}$ be an ideal of $\mathfrak{X}_{t}$ for $t \in T$. Then $\bigcup_{t \in T} I_{t}$ is an ideal of $\mathfrak{X}$. Conversely, every ideal of $\mathfrak{X}$ is of this form.

Proof. Let $I=\bigcup_{t \in T} I_{t}$. Of course, $0 \in I$. Let $x * y \in I$ and $y \in I$. If $x \in X_{t}$ and $y \in X_{u}$, where $t \neq u$, then $x=x * y \in I$. Suppose that $x, y \in X_{t}$. Then $x * y, y \in I_{t}$. Since $I_{t}$ is an ideal of $\mathfrak{X}_{t}$, we conclude that $x \in I_{t}$. Hence $x \in I$, and consequently, $I \in \operatorname{Id}(\mathfrak{X})$.

Now let $I$ be an ideal of $\mathfrak{X}$. It is easy to see that $I_{t}:=I \cap X_{t} \in \operatorname{Id}\left(\mathfrak{X}_{t}\right)$ for $t \in T$. We have $I=I \cap \bigcup_{t \in T} X_{t}=\bigcup_{t \in T} I \cap X_{t}=\bigcup_{t \in T} I_{t}$.

Example 3.12. Consider the pseudo-BCH-algebras $\mathfrak{X}_{1}, \mathfrak{X}_{2}$, and $\mathfrak{X}$, which are described in Example 2.6. We know that $\operatorname{Id}\left(\mathfrak{X}_{1}\right)=\left\{\{0\},\{0, a\},\{0, b\}, X_{1}\right\}$ (by Example 3.5). It is easy to check that $\operatorname{Id}\left(\mathfrak{X}_{2}\right)=\left\{\{0\},\{0,3\}, X_{2}\right\}$. Applying Theorem 3.11, we get $\operatorname{Id}(\mathfrak{X})=\left\{\{0\},\{0, a\},\{0, b\}, X_{1},\{0,3\},\{0,3, a\}\right.$, $\left.\{0,3, b\}, X_{1} \cup\{3\}, X_{2}, X_{2} \cup\{a\}, X_{2} \cup\{b\}, X\right\}$.

Cen $\mathfrak{X}$ is a subalgebra of $\mathfrak{X}$ (see Proposition 2.8) but it may not be an ideal. For example, let $Y=\{0, a, b, c, d, e, f, g, h\}$ and $\mathfrak{Y}=(Y ; *, \diamond, 0)$ be the pseudo-BCH-algebra given in Example 2.5. Then Cen $\mathfrak{Y}=\{0, d, e, f, g, h\}$. It is easy to see that Cen $\mathfrak{Y}$ is not an ideal of $\mathfrak{Y}$. Now we establish conditions for the set $\mathrm{Cen} \mathfrak{X}$ to be an ideal of a pseudo-BCH-algebra $\mathfrak{X}$.

Theorem 3.13. Let $\mathfrak{X}$ be a pseudo-BCH-algebra. The following statements are equivalent:

(i) Cen $\mathfrak{X}$ is an ideal of $\mathfrak{X}$.

(ii) $x=(x * a) *(0 * a)$ for $x \in X, a \in$ Cen $\mathfrak{X}$.

(iii) For all $x \in X, a \in$ Cen $\mathfrak{X}, x * a=0 * a$ implies $x=0$.

(iv) For all $x \in \mathrm{K}(\mathfrak{X}), a \in \mathrm{Cen} \mathfrak{X}, x * a=0 * a$ implies $x=0$.

Proof. (i) $\Longrightarrow$ (ii): Write $I=$ Cen $\mathfrak{X}$, and suppose that $I$ is an ideal of $\mathfrak{X}$. Let $x \in X$ and $a \in I$. By (pBCH-2) and (pBCH-1),

$((x * a) *(0 * a)) \diamond x=((x * a) \diamond x) *(0 * a)=((x \diamond x) * a)) *(0 * a)=(0 * a) *(0 * a)=0$, and hence

$$
(x * a) *(0 * a) \leqslant x .
$$


Using (pBCH-2) and (a1), we obtain

(6) $\quad(x \diamond((x * a) *(0 * a))) * a=(x * a) \diamond((x * a) *(0 * a)) \leqslant 0 * a$.

By (3), $0 * a \in I$. From (6) and Proposition 3.2 we conclude that

$$
(x \diamond((x * a) *(0 * a))) * a \in I .
$$

Since $a \in I$, by the definition of ideal we deduce that

$$
x \diamond((x * a) *(0 * a)) \in I .
$$

Applying (a6) and Proposition 2.7, we get

$0 *((x * a) *(0 * a))=(0 *(x * a)) \diamond(0 *(0 * a))=(a * x) \diamond a=(a \diamond a) * x=0 * x$.

Then $0 *(x \diamond((x * a) *(0 * a)))=(0 * x) *(0 * x)=0$, and hence

$$
x \diamond((x * a) *(0 * a)) \in \mathrm{K}(\mathfrak{X}) .
$$

From this and $(7)$ we have $x \diamond((x * a) *(0 * a)) \in I \cap \mathrm{K}(\mathfrak{X})=\{0\}$ (see (4)), that is, $x \diamond((x * a) *(0 * a))=0$. Therefore

$$
x \leqslant(x * a) *(0 * a) .
$$

By (5) and (8) we obtain $x=(x * a) *(0 * a)$.

(ii) $\Longrightarrow$ (iii): Let $x \in X, a \in$ Cen $\mathfrak{X}$, and $x * a=0 * a$. Then $x=$ $(x * a) *(0 * a)=(x * a) *(x * a)=0$.

(iii) $\Longrightarrow$ (iv) is obvious.

(iv) $\Longrightarrow$ (i): To prove that Cen $\mathfrak{X}$ is an ideal, let $a, x * a \in$ Cen $\mathfrak{X}$. Observe that $x \diamond(0 *(0 * x)) \in \mathrm{K}(\mathfrak{X})$. By $(\mathrm{a} 6)$ and $(\mathrm{a} 4), 0 *[x \diamond(0 *(0 * x))]=$ $(0 * x) *(0 *(0 *(0 * x)))=(0 * x) *(0 * x)=0$, and hence

$$
x \diamond(0 *(0 * x)) \in \mathrm{K}(\mathfrak{X}) .
$$

We have

$$
\begin{aligned}
x * a & =0 *(0 *(x * a)) & & {[\text { since } x * a \in \text { Cen } \mathfrak{x}} \\
& =(0 *(0 * x)) *(0 *(0 * a)) & & {[\text { by }(\text { a } 5) \text { and }(\mathrm{a} 6)] } \\
& =(0 *(0 * x)) * a . & & {[\text { since } a \in \text { Cen } \mathfrak{X}] }
\end{aligned}
$$

Then by (pBCH-2) and (pBCH-1),

$[x \diamond(0 *(0 * x))] * a=(x * a) \diamond(0 *(0 * x))=[(0 *(0 * x)) * a] \diamond(0 *(0 * x))=0 * a$,

that is,

$$
[x \diamond(0 *(0 * x))] * a=0 * a .
$$

Applying (iv) we get $x \diamond(0 *(0 * x))=0$. Hence $x \leqslant 0 *(0 * x)$. By (a3) and (a1), $0 *(0 * x)=0 *(0 \diamond x) \leqslant x$, and therefore $x=0 *(0 * x)$. From this $x \in$ Cen $\mathfrak{X}$. Thus Cen $\mathfrak{X}$ is an ideal of $\mathfrak{X}$. 
We also have theorem analogous to Theorem 3.13.

Theorem 3.14. Let $\mathfrak{X}$ be a pseudo-BCH-algebra. The following statements are equivalent:

(i) Cen $\mathfrak{X}$ is an ideal of $\mathfrak{X}$.

(ii) $x=(x \diamond a) \diamond(0 \diamond a)$ for $x \in X, a \in$ Cen $\mathfrak{X}$.

(iii) For all $x \in X, a \in$ Cen $\mathfrak{X}, x \diamond a=0 \diamond a$ implies $x=0$.

(iv) For all $x \in \mathrm{K}(\mathfrak{X}), a \in \mathrm{Cen} \mathfrak{X}, x \diamond a=0 \diamond a$ implies $x=0$.

\section{REFERENCES}

[1] Dudek, W. A., Thomys, J., On decompositions of BCH-algebras, Math. Japon. 35 (1990), 1131-1138.

[2] Dudek, W. A., Zhang, X., On atoms in BCC-algebras, Discuss. Math. Algebra Stochastic Methods 15 (1995), 81-85.

[3] Dudek, W. A., Jun, Y. B., Pseudo-BCI-algebras, East Asian Math. J. 24 (2008), 187-190.

[4] Dudek, W. A., Zhang, X., Wang, Y., Ideals and atoms of BZ-algebras, Math. Slovaca 59 (2009), 387-404.

[5] Dudek, W. A., Karamdin, B., Bhatti, S. A., Branches and ideals of weak BCCalgebras, Algebra Colloq. 18 (Special) (2011), 899-914.

[6] Dvurečenskij, A., Pulmannová, S., New Trends in Quantum Structures, Kluwer Acad. Publ., Dordrecht; Ister Science, Bratislava, 2000.

[7] Dymek, G., Atoms and ideals of pseudo-BCI-algebras, Comment. Math. 52 (2012), 73-90.

[8] Dymek, G., On pseudo-BCI-algebras, Ann. Univ. Mariae Curie-Skłodowska Sect. A 69 (1) (2015), 59-71.

[9] Georgescu, G., Iorgulescu, A., Pseudo-BCK algebras: an extension of BCK algebras, in Proc. of DMTCS'01: Combinatorics, Computability and Logic, 97-114, Springer, London, 2001.

[10] Hu, Q. P., Li, X., On BCH-algebras, Math. Seminar Notes 11 (1983), 313-320.

[11] Imai, Y., Iséki, K., On axiom systems of propositional calculi XIV, Proc. Japan Acad. 42 (1966), 19-22.

[12] Iséki, K., An algebra related with a propositional culculus, Proc. Japan Acad. 42 (1966), 26-29.

[13] Iorgulescu, A., Algebras of Logic as BCK-algebras, Editura ASE, Bucharest, 2008.

[14] Kim, K. H., Roh, E. H., The role of $A^{+}$and $A(X)$ in $B C H$-algebras, Math. Japon. 52 (2000), 317-321.

[15] Kim, Y. H., So, K. S., On minimality in pseudo-BCI-algebras, Commun. Korean Math. Soc. 27 (2012), 7-13.

[16] Lee, K. J, Park, C. H., Some ideals of pseudo-BCI-algebras, J. Appl. Math. Inform. 27 (2009), 217-231.

[17] Meng, J., Xin, X. L., Characterizations of atoms in BCI-algebras, Math. Japon. 37 (1992), 359-361.

[18] Walendziak, A., Pseudo-BCH-algebras, Discuss. Math. Gen. Algebra Appl. 35 (2015), $1-15$.

[19] Walendziak, A., Wojciechowska-Rysiawa, M., Fuzzy ideals of pseudo-BCH-algebras, Mathematica Aeterna 5 (2015), 867-881. 
Andrzej Walendziak

Institute of Mathematics and Physics

Faculty of Science

Siedlce University of Natural Sciences and Humanities

3 Maja 54, PL-08110 Siedlce

Poland

e-mail: walent@interia.pl

Received January 17, 2016 